\title{
Movilidad laboral urbana y Covid-19: los casos de tres zonas metropolitanas de México
}

\section{Urban labor mobility and Covid -19: the cases of three metropolitan areas of Mexico}

\author{
José Antonio Soberón Mora \\ Juan Gabino González Becerril \\ Rodrigo Aguilar Zepeda \\ Eduardo Andrés Sandoval Forero
}

\author{
Centro de Investigación y Estudios Avanzados de la Población \\ de la Universidad Autónoma del Estado de México
}

\section{Resumen}

El documento tiene como objetivo evaluar la relación entre la movilidad laboral y el contagio como las defunciones causadas por Covid-19 entre marzo y octubre de 2020. Se seleccionan tres Zonas Metropolitanas ubicadas en el centro y occidente de México. Para realizar esta relación nos apoyamos en la Encuesta Intercensal de 2015 y la información derivada de la base de datos de la Secretaría de Salud en México con indicadores diarios sobre el padecimiento. Al procesar los datos de ambas fuentes de información, se obtienen valores positivos al aplicar una correlación de Pearson con la mayoría de las variables introducidas en el análisis. Los casos más destacados tienen que ver con las personas que se trasladan a laborar dentro del mismo municipio de residencia, todos muestran valores significativos. Como segundo resultado a destacar (con pocas probabilidades de contagiarse o fallecer), es importante el papel que juega la población que se traslada a laborar en bicicleta.

Palabras clave: Movilidad laboral, Covid-19.

\section{Abstract}

The document aims to evaluate the relationship between labor mobility and contagion such as deaths caused by Covid-19 between March and October 2020. Three Metropolitan Zones located in the center and west of Mexico are selected. To carry out this relationship, we rely on the 2015 Intercensal Survey and the information derived from the database of the Ministry of Health in Mexico with daily indicators on the condition. When processing the data from both sources of information, positive values are obtained by applying a Pearson correlation with most of the variables introduced in the analysis. The most prominent cases have to do with people who move to work within the same municipality of residence, all show significant values. As a second result to highlight (with little probability of being infected or dying), the role played by the population that travels to work by bicycle is important.

Keywords: Labor mobility, Covid-19. 


\section{INTRODUCCIÓN}

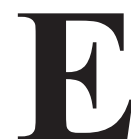

n diciembre de 2019 la Organización Mundial de la Salud (OMS) informó de la existencia del coronavirus SARS-CoV-2 causante del Covid-19, además de comunicar de la existencia de casos de neumonía vírica en Wuhan en la República Popular China.

El anuncio se acompañó de noticias que mostraron el inminente avance del nivel de contagio y del poder de diseminación mucho mayor en las ciudades en las cuales según la Naciones Unidas se concentraba 90 por ciento de contagios. ${ }^{1}$ De forma similar, se ha difundido entre los sectores de la población, principalmente en las zonas metropolitanas, que las regiones de mayor vulnerabilidad son las zonas rurales, debido principalmente a problemas de acceso a los servicios de salud requeridos para la atención de este padecimiento (Tabla 1).

Tabla 1: Total de contagios y fallecimientos por Covid-19 en México al 20 de octubre de 2020, 2015

\begin{tabular}{lrrr}
\hline & Fallece & Contagiado & Total \\
\hline Zona Metropolitana del Valle de México & & & \\
Positivo SARS-CoV-2 & 20,127 & 191,895 & 212,022 \\
No positivo SARS-CoV-2 & 5,381 & 330,880 & 336,261 \\
Zona Metropolitana de Guadalajara & & & \\
Positivo SARS-CoV-2 & 2,530 & 19,050 & 21,580 \\
No positivo SARS-CoV-2 & 967 & 28,392 & 29,359 \\
Zona Metropolitana de Toluca & & & \\
Positivo SARS-CoV-2 & 1,870 & 12,244 & 14,114 \\
No positivo SARS-CoV-2 & 409 & 18741 & 19,150 \\
\hline
\end{tabular}

Fuente: Secretaría de Salud (México).

Sobre este tema, los estudios se han centrado en el volumen de contagios, en la mortalidad, así como en las características demográficas de la población afectada, ${ }^{2}$ y dado nuestro interés nos centraremos en el análisis de cohorte metropolitano. En este sentido, el texto se organiza de la siguiente forma: medidas adoptadas para mitigar el contagio por Covid-19, antecedentes, la movilidad cotidiana en las Zonas Metropolitanas seleccio-

1 Véase en https://coronavirus.onu.org.mx/las-ciudades-concentran-el-90-de-los-casos-de-covid-19 (30/01/2021).

2 Hernández Bringas, Héctor, Covid-19 en México: un perfil sociodemográfico, consultado en https://repositorio.cepal.org/handle/11362/46557 (30/01/2021). 
nadas, movilidad laboral, metodología y fuentes de información, resultados y conclusiones.

\section{Medidas adoptadas Para mitigar el CONTAgio POR Covid-19}

Algunas de las medidas de protección promueven el distanciamiento físico; el uso de mascarilla, "especialmente cuando no se pueda mantener el distanciamiento" (OMS, 2020) mantener las habitaciones ventiladas, evitar aglomeraciones, así como el contacto estrecho con otras personas. La formulación de estas medidas ha buscado mitigar la transmisión del virus al tener la capacidad de transmitirse a través de gotículas respiratorias con un diámetro de cinco a diez micrómetros $(\mu \mathrm{m})(1000$ micrómetros $=1$ milímetro).

Otras de las medidas llevadas a cabo, por el gobierno mexicano en este caso, han consistido en limitar el acceso a los servicios de transporte de la población bajo su jurisdicción, esto con la intención de limitar el contacto de las personas que se trasladan en las ciudades por diversos motivos. Desde entonces y en el transcurso del año 2020 se han tomado medidas tendientes a mitigar los niveles de contagio del virus, las medidas que se describen se insertan en un nivel estatal y limitan la distribución de la población respectiva en zonas metropolitanas (Tabla 2). Los casos que se exponen no pretenden ser exhaustivos de todas las medidas que se han tomado y únicamente son demostrativos de la modalidad de contagio por las variables que se analizan. Sin embargo, dichas medidas han sido cuestionadas a tal grado que algunos observadores en el mundo han argumentado que el gobierno de México ha inducido al contagio masivo o la inmunidad de rebaño.

\section{Estado de México}

De esta manera tenemos el caso del Estado de México, en donde el 21 de abril de 2020 y en lo que respecta al transporte público, determinó que el Mexibús y el Mexicable operarían al 50 por ciento de su capacidad para mantener la sana distancia, la restricción también aplicaría para el transporte público concesionado, es decir, cada autobús sólo podría circular si va a la mitad de su capacidad. En el Valle de Toluca se aplicaría el programa vehicular el "Hoy No Circula".

En este caso, el mexibús y el mexicable operan hacia el norte de la Zona Metropolitana del Valle de México y una parte del transporte concesionado en la Zona Metropolitana de Toluca, ambas regiones pertenecientes al Estado de México. Aquí por la contigüidad geográfica los gobiernos de la 
Ciudad de México y de la entidad mexiquense comparten una política común de prevención, pero también la diferencia del médico en tu domicilio y el llenado de tanques de oxígeno medicinal gratuito entre otros que el Gobierno del Estado de México no implementa. ${ }^{3}$

Tabla 2: fases de la contingencia, medidas preventivas, 2015

\begin{tabular}{|c|c|c|}
\hline Fase 1 & Fase 2 & Fase 3 \\
\hline $\begin{array}{l}\text { Número reducido } \\
\text { de infectados }\end{array}$ & Distanciamiento social & Distanciamiento social \\
\hline \multirow[t]{7}{*}{$\begin{array}{l}\text { No hay medidas } \\
\text { estrictas de sanidad pero } \\
\text { sí reducción del contacto } \\
\text { físico, lavarse las manos, } \\
\text { supervisión de casos } \\
\text { detectados }\end{array}$} & $\begin{array}{l}\text { Evitar el saludo de mano, } \\
\text { beso y abrazo }\end{array}$ & $\begin{array}{l}\text { Difusión intensiva y } \\
\text { permanente de las } \\
\text { medidas de mitiga- } \\
\text { ción, de los síntomas } \\
\text { y signos de alarma de } \\
\text { Covid-19 }\end{array}$ \\
\hline & $\begin{array}{l}\text { Medidas de higiene } \\
\text { básicas }\end{array}$ & $\begin{array}{l}\text { Suspender contacto } \\
\text { con personas diagnos- } \\
\text { ticadas o con sospecha } \\
\text { de la enfermedad }\end{array}$ \\
\hline & Estornudo de etiqueta & $\begin{array}{l}\text { Suspensión indefini- } \\
\text { da de actividades no } \\
\text { esenciales y eventos } \\
\text { ante brotes }\end{array}$ \\
\hline & $\begin{array}{l}\text { Suspensión temporal de } \\
\text { actividades no esenciales } \\
\text { y escolares }\end{array}$ & $\begin{array}{l}\text { Filtros sanitarios en la } \\
\text { entrada de inmuebles }\end{array}$ \\
\hline & $\begin{array}{l}\text { Filtros sanitarios en la } \\
\text { entrada de inmuebles }\end{array}$ & \\
\hline & $\begin{array}{l}\text { Suspensión de eventos } \\
\text { masivos en espacios } \\
\text { abiertos }\end{array}$ & \\
\hline & $\begin{array}{l}\text { Protección y cuidado de } \\
\text { grupos vulnerables }\end{array}$ & \\
\hline
\end{tabular}

Fuente: IMSS (2020).

\footnotetext{
3 Patiño Fierro, Martha Patricia y Cruz Reyes, Gerardo, (2020), "Las medidas adoptadas por las entidades federativas ante la emergencia del Covid-19", Cuaderno de Investigación No. 7, DGDyP/IBD, CDMX, 162 pp., consultado en http://bibliodigitalibd.senado.gob.mx/bitstream/handle/123456789/4856/CuadernoDeInvestigaci\%C3\%B3n_7\%20\%28F\%29.pdf?sequence=1\&isAllowed $=y(31 / 01 / 2021)$.
} 


\section{Ciudad de México (Zona Metropolitana del Valle de México)}

En la Gaceta oficial de la Ciudad de México del 1 de abril de 2020 de establecen una serie de acciones extraordinarias ante la contingencia del Covid-19 en donde se suspenden todas las actividades no esenciales durante un período de 30 días. Esta publicación es ilustrativa porque en el capítulo VI se exhorta a la población de la Ciudad de México, entre otras medidas, a cumplir con el resguardo domiciliario correspondiente a la fecha marcada y un punto importante lo precisa cuando "Se entiende como resguardo domiciliario correspondiente a la limitación voluntaria de movilidad, permaneciendo en el domicilio particular o sitio distinto al espacio público, el mayor tiempo posible" (Gaceta Oficial, 2020: 4).

En esta demarcación que pertenece a la Zona Metropolitana del Valle de México, el 20 de mayo de 2020 el secretario de movilidad, Andrés Lajous coincidió en que al levantarse las restricciones y retomar las actividades, podría haber tres posibilidades para que la gente se mueva y evite las multitudes del transporte público: que se aumenten los viajes en auto particular, en motocicleta y bicicleta.

\section{Guadalajara}

En la Zona Metropolitana de Guadalajara la operación del transporte público se suspendería a partir de las 20:59 horas, reiniciando a las 05:30 horas del día siguiente. Después de las 20:59 horas únicamente se permitirá la circulación de unidades para bajar a los pasajeros, terminar su recorrido y arribar a su zona de pernocta.

Los servicios de empresas de redes de transporte o plataformas (Didi, Uber), solo podrán aceptar servicios de lunes a viernes de 05:30 horas hasta las 19:59 horas podrán aceptar su último viaje. ${ }^{4}$

Otras entidades que han tomado medidas respecto a la movilidad se han enfocado a la circulación de los medios terrestres en donde se especifican horarios de funcionamiento como principal medida. ${ }^{5}$

\footnotetext{
${ }^{4}$ El metro de la entidad se localiza en la Ciudad de Guadalajara, pero por concentrarse en el municipio del mismo nombre no es posible realizar el análisis sugerido.

${ }_{5}$ Monterrey. En esta entidad se implementan medidas y horarios en el transporte público de la ciudad de Nuevo León a partir del 19 de mayo de 2020, ante la contingencia sanitaria.

En lo que respecta a las rutas urbanas y suburbanas, específicamente la ruta express y transmetro, el horario sería el habitual y tendrán un espacio de paso de 15 minutos entre cada unidad de transporte. El horario sería corrido como de costumbre de 4:00 horas a 00:00 horas. En las "horas valle" 09:30 horas a 16:00 horas el servicio se ofrecerá con una frecuencia de paso más espaciada entre unidades de un promedio de 30 minutos.

Chihuahua. En un evento más reciente y en Chihuahua, el cuatro de noviembre de 2020 el gobernador de esta entidad Javier Corral, anunció un conjunto de medidas restrictivas ante el aumento de casos de Covid-19. Entre estas se estableció que el servició de transporte público estaría
} 


\section{Antecedentes}

A principios del año 2020 ya era de dominio público el impacto que por motivo de contagio causaba la transmisión de SARS-CoV-2 a nivel mundial. Para el 15 de abril, se había reportado una cifra que superaba los 1.8 millones de casos y más de ciento diez mil decesos por Covid-19 (Jarvis et al., 2020; ECDC, 2020).

Una de las principales vías de transmisión del SARS-CoV-2 es la propagación por gotículas que requiere un contacto estrecho. En un intento por mitigar la pandemia de Covid-19, muchos países habían adoptado políticas de distanciamiento físico sin precedentes (Acaps, 2020). De esta manera, para finales de mayo de 2020 los gobiernos de distintos países habían implementado medidas que instruían a la población a quedarse en casa y evitar salir excepto para trabajos esenciales. Estas medidas de distanciamiento físico han tenido como objetivo mitigar el impacto de la pandemia de Covid-19. Sin embargo, no se había aclarado en qué medida estas disposiciones reducirían el número de contactos $\mathrm{y}$, por tanto, la transmisión. Como parte de un interés por los resultados de estas normas, una encuesta realizada en dos ciudades chinas, Wuhan y Shanghai, en febrero de 2020, cuantificó su impacto en los patrones de contacto de las personas durante la pandemia, en donde parte de las contribuciones apoyan la existencia de los contagios asintomáticos en la población de 0 a 14 años y el alto riesgo en población con más de 65 años (Zhang, J, et al., 2020).

A partir de las medidas de distancia física se realizaron trabajos sobre la relación que pudiese tener el Covid-19 y la movilidad a nivel mundial, esta última se ha explorado principalmente a partir de los efectos que la pandemia ha tenido y tendrá sobre el transporte, principalmente el urbano. Bajo este panorama, los proyectos revisados para este documento, en su mayoría abordan la relación entre pandemia y transporte urbano. En este

\footnotetext{
restringido los fines de semana. Cabe notar que la restricción afectaba menos al transporte de la población económicamente activa como se verá. Concretamente, los dos fines de semana posteriores a este anuncio, el 7 y 8 de noviembre y el 14 y 15 del mismo mes sólo operarían rutas de transporte público que tuvieran como destino unidades hospitalarias y sólo se haría parada en esos puntos. Se aprecia que las medidas tomadas habrían tendido a facilitar el transporte de la población vinculada procesos de salud ya sea desde el punto de vista de la población usuaria de los servicios de salud, del personal médico ligado a este rubro o de los servicios asociados. Los servicios de empresas de redes de transporte o plataformas sólo podrían aceptar servicios de lunes a viernes con horario de las 6:00 de la mañana a las 20:00 horas quedando imposibilitadas de circular el fin de semana, esto es el sábado y el domingo, con excepción de traslados cuyo origen o destino sea una farmacia o un centro de salud u hospital. Estas medidas se tomaron con una ocupación hospitalaria de 90 por ciento (AP, 2020).

Otras medidas que se han tomado con la finalidad de disminuir los niveles de contagio incluyen restricciones a la actividad comercial. Al cierre de este documento las medidas que afectaban al desempeño económico estaban activas. Bajo estas condiciones, no suponemos que se excluyan los efectos de unas medidas con otras.
} 
aspecto, la mayor parte de los estudios realizados para medir el impacto entre ambas variables, la transmisión de la epidemia y el transporte urbano se dirigen a examinar factores de dependencia en donde el transporte es la variable explicativa del contagio.

Si bien, no obstante la preeminencia de las temáticas que se enfocan a explorar los resultados de las restricciones en contacto físico, en distintos países se han presentado resultados de investigaciones que se han abocado a captar la distancia física y los efectos sobre el transporte público pero a partir de variables como su percepción, la frecuencia de uso, las posibilidades de retomar su uso a partir de la reducción de las restricciones, la seguridad percibida etc. Otras investigaciones han explorado también el efecto de la pandemia sobre los entornos vecinales o comunales. Una condición más específica se presentó al tomar en cuenta los contactos no domésticos $\mathrm{y}$ aquellos que se presentan en ubicaciones fuera del contexto familiar y vecinal (Feehan y Mahmud, 2020; Jarvis et al., 2020).

Investigaciones posteriores muestran que para entornos cerrados como los vehículos de transporte público, el uso adecuado de máscaras faciales ha reducido significativamente la probabilidad de contagio (Tarachini y Cats, 2020), pero a pesar de estas aportaciones son pocos los informes que proporcionan evidencia de la evolución de los contagios a partir de medidas aplicadas en el transporte público.

Una contribución posterior realizada en el Reino Unido reportó una disminución en la transmisión de Covid-19 como respuesta al distanciamiento físico. Los resultados de este estudio se enfocan principalmente en el contacto entre personas en ambientes cerrados y no obstante su contribución principal perfila el contacto bajo diversas condiciones como son las laborales, las escolares o las actividades de distracción, el contacto en transporte público también es reportado. Los resultados contribuyeron a sostener que el distanciamiento entre personas a partir de las modalidades que se han mencionado contribuía a disminuir los contagios de manera poco consistente. Los autores señalaron que no obstante era menor la evidencia mostrada por las medidas tomadas en contra del contagio, se consideraba todavía limitado el tiempo transcurrido para esperar resultados más concluyentes (Jarvis et al., 2020).

Los resultados de las investigaciones revisadas y que recurren al distanciamiento físico como medida precautoria, concluyen que esta medida se encuentra asociada a la disminución de los niveles de contagio de Covid-19. Se reconoce también que al momento de realizar las investigaciones respectivas, era prematuro concluir el efecto de las medidas toma- 
das y que se requeriría de más tiempo para tomar alguna determinación con base más sólida.

\section{LA MOVILIDAD COTIDIANA EN LAS ZONAS METROPOLITANAS}

La población de México está en constante movilidad y sobre todo en ascenso para la mayoría de zonas metropolitanas si se considera el criterio de la movilidad intrametropolitana. López (2016) en sus estimaciones sobre la movilidad espacial por motivos de trabajo perfilada en todo el país, observó que la tendencia de la movilidad cotidiana en México abarca cada vez a mayor población. Los resultados agregados nacionales indican que: i) La población que se desplaza a otro municipio distinto de su residencia pero que trabaja en la misma entidad, osciló de 16.6 por ciento en 2000 (de 35 millones de personas ocupadas), a 14.5 por ciento en 2010 (de 43 millones de ocupados) y a 15 por ciento en 2015 (44.6 millones de ocupados). ii) Los movimientos que atraviesan otra entidad reportan una proporción estable (5.1 por ciento en $2000,5.4$ por ciento en 2010 y 5.4 por ciento en 2015). Como se observa, la población que se traslada al trabajo en datos absolutos como relativos aumenta progresivamente.

La movilidad de personas por trabajo entre un municipio y otro es conocida técnicamente y en inglés como commuters, los cuales pueden variar a lo largo del territorio nacional, porque algunos pueden presentar este intercambio muy bajo derivado de la oferta y demanda del mercado de trabajo. Pero, otros tantos sí lo tienen y se estima que 796 de esos municipios están por arriba de la media nacional (18.8 por ciento) (UNAM, 2015).

Por ejemplo, en la Zona Metropolitana del Valle de México (ZMVM), se estima que entre el año 2000 y 2010, los commuters pasaron de 3.3 a 3.6 millones de personas. Otro ejemplo, fue que el número de commuters en la Zona Metropolitana de Guadalajara (ZMGDL) que paso de $304 \mathrm{mil}$ a 534 mil entre 2000 y 2015 (UNAM, 2015). Para la Zona Metropolitana de Toluca (ZMT) los ocupados con movilidad laboral fue de 44 mil 825 en 2007, 72 mil 011 personas en 2010 y los migrantes intrametropolitanos en Toluca 51 mil (Hoyos et al., s/f; Granados et al., 2017; Romo, 2018), la última estimación es muy parecida a la de Hernández y Benítez (2017) cifrada en 50, 750 personas que migraron dentro de la ZMT asociados al trabajo con uso intensivo del conocimiento.

Camacho (2015) distingue relaciones funcionales en la ZMT con base a flujos origen-destino, considerando tres ámbitos de flujos. De un total de 44,825 encuestas origen-destino aplicadas en 2007, el primer ámbito, considerando el 
intra-metropolitano como principal origen y destino del total de desplazamientos (72 por ciento); de ellos se realizan en el área urbana continua 80 por ciento cuyo nodo principal es el municipio de Toluca, el resto de municipios generaba el otro 20 por ciento. Es un patrón centro-periferia donde el centro mantiene supremacía. El segundo ámbito de los movimientos, desde la ZMT al Estado de México, muestra interrelaciones externas con 67 municipios cuyo principal motivo de traslado es el de trabajo (16 por ciento del total). El tercer ámbito, la relación de la ZMT con otras entidades del país, muestra vinculación con 27 estados (12 por ciento del total) donde destacan las relaciones con Ciudad de México y estado de Michoacán, aunque también aparecen entidades de mayor distancia. Según los flujos de los tres ámbitos, la ZMT mantiene interacción por razones económicas con otras ciudades del sistema nacional, ello devela que las relaciones diarias son extensas que no se circunscriben sólo al espacio metropolitano, ni al espacio estatal sino también con el territorio abierto, aunque la organización interna sigue siendo centro-periferia (Hoyos et al., (s/f).

\section{LA MOVILIDAD LABORAL}

La movilidad usualmente se realiza en forma periódica, diario, y excluye los días de descanso que pueden ser intercalados dentro de un programa semanal. Los motivos para el traslado son diversos: los laborales, estudio, esparcimiento y de servicios. En nuestro caso, nos centramos específicamente por motivos laborales que son la expresión de la oferta y demanda ahora en tiempos de la pandemia. Ramírez (2015) lo indica de manera más precisa al elaborar la distinción entre movilidad laboral (que también es cotidiana) y movilidad por otros asuntos (cotidiana). La primera se define por la posición que se tiene en el trabajo y, por lo tanto, se relaciona con el acceso laboral a los mercados regionales metropolitanos. La segunda que denomina exclusivamente movilidad cotidiana, integra a las amas de casa, los estudiantes y todos aquellos agentes cuyo motivo principal de desplazamiento no es de carácter laboral (Ramírez, 2015: 26).

Para este proyecto, entenderemos por movilidad laboral a la población que se traslada de un lugar a otro, regularmente desde su lugar de residencia hasta su lugar de trabajo, con el objeto de desempeñar sus actividades laborales. Esta definición no puede excluir a la definición de la población a partir de su lugar de residencia por dos razones: i) el movimiento debe estar referido una función que incluya origen-destino y por lo tanto retorno a una residencia habitual; ii) la fuente de información consultada integra sus registros con base a una residencia habitual, modalidad que permite armar los desplazamientos correspondientes de la población involucrada. 
Al precisar la dinámica de la movilidad en las zonas metropolitanas, en este documento y para fines metodológicos se define además de lo establecido, como el traslado de la Población Económicamente Activa-Ocupada. Así, la movilidad es la estrategia utilizada por la población para organizar su actividad diaria y tiene como objetivo el acceso al lugar de trabajo (Potrykowski y Taylor, 1984). Adicionalmente los sistemas de transporte se refieren a la infraestructura, que agrupa a las redes de transporte como a las instalaciones requeridas para su funcionamiento, los flujos de transporte, conformados por el tráfico y los servicios de transporte, componentes organizativos de los trayectos y recorridos (Potrykowski y Taylor, 1984).

\section{METODOLOGÍA Y FUENTES DE INFORMACIÓN}

Uno de los retos en la investigación científica, siempre será la de combinación de fuentes de información. Se procura que la diferencia de fechas sea mínima, al a vez que se busca sincronicidad temporal de tal manera que permita reducir el número de condiciones adversas que podrían influir en los resultados obtenidos. El documento que aquí presentamos considera esta condición, es decir, lo más cercano son cinco años de diferencia entre la Encuesta Intercensal y los datos del Covid-19 de la Secretaría de Salud. Así, el proyecto Movilidad Laboral Urbana y Covid-19 aquí presentado, recupera el comportamiento registrado de la enfermedad SARS-CoV-2, que son los infectados como de los fallecidos por este padecimiento y su relación con los traslados que se registran de las personas que trabajan en las zonas urbanas, esto a partir del examen de tres zonas metropolitanas del país ya mencionadas.

Se parte del supuesto de que, a mayor cantidad de traslados por motivos laborales dentro de las zonas metropolitanas citadas y por los medios de transporte mencionados, se incrementará el número de infectados y fallecidos por Covid-19. Para ello se recurre en primer lugar a la información captada por la Encuesta Intercensal realizada por INEGI en el año 2015 (EIC 2015), misma que cuenta con cobertura nacional y que capta información sobre los sujetos que trabajan, específicamente capta a la Población Económicamente Activa Ocupada (PEA-O), la cual es registrada como la población con 12 o más años cumplidos que trabajó o buscó trabajo la semana anterior a la del levantamiento. La EIC 2015 se aplicó a nivel vivienda y capta la información de todos los residentes de los hogares seleccionados con una precisión de estimación reportada por segmento. Para precisar los traslados respecto al medio utilizado también se utilizan variables como ubicación del lugar de trabajo y el tiempo de traslado. Cabe precisar que el 
interés específico sobre el tipo de traslado para este proyecto se ubica en el transporte público, aspecto contemplado en la parte de antecedentes de este documento y que permite precisar el planteamiento hipotético.

Lo anterior, implica operacionalizar el uso de las bases de datos para la comprobación de la hipótesis lo cual consta de dos pasos. El segundo de ellos es contabilizar las defunciones y los infectados por Covid-19 a partir de los registros publicados por la Secretaría de Salud de manera continua y a partir del mes de abril del año 2020. Estos registros son de carácter público y se encuentran alojados en la página respectiva de la Secretaría de Salud. El formato para acceder a esta fuente de información es mediante la gestión de cualquier manejador de bases de datos, para este caso, como en el siguiente, se recurre a la versión 25 de SPSS. Dado que ambas bases utilizadas captan el municipio respectivo de cada entidad; los acontecimientos ocurridos con Covid-19 por el lado de la encuesta de Salud y la residencia de los encuestados en la EIC 2015 por el otro, se procede entonces a estimar si la cantidad de personas que laboran en un municipio determinado se encuentra relacionado con la cantidad de personas infectadas o fallecidas por Covid-19. ${ }^{6}$

En términos simples, la EIC 2015, cuyas muestras se encuentran integradas por los sujetos que radican en las entidades de Hidalgo, Jalisco, Estado de México y Ciudad de México, se componen por quienes radican específicamente en los municipios que forman las zonas metropolitanas analizadas. Esta población se compone exclusivamente de la Población Económicamente Activa Ocupada (PEA-O). ${ }^{7}$

Con base en dichas fuentes de información se procede a cuantificar la relación por medio de ejecutar una $r$ de Pearson en su modalidad de correlación bivariada misma que consiste en la fórmula (Glass y Stanley, 1974):

$$
r_{x y}=\frac{n \sum X_{i} Y_{i}-\left(\sum X_{i}\right)\left(\sum Y_{i}\right)}{\sqrt{\left[n \sum X_{i}^{2}-\left(\sum X_{i}\right)^{2}\right]\left[n \sum Y_{i}^{2}-\left(\sum Y_{i}\right)^{2}\right]}} .
$$

\section{Resultados}

El análisis de las respectivas correlaciones bivariadas implica tener presente dos aspectos: a) el resultado de las correlaciones es con una parte de la población, cada opción es excluyente y supone el comportamiento bajo

\footnotetext{
${ }^{6}$ Debe aclararse que a la fecha de envío de este documento todavía no se pueden consultar los resultados del censo del año 2020 lo que provoca un desfase de cinco años en relación con las fuentes consultadas.

${ }^{7}$ Se hace referencia a tres zonas metropolitanas y a sus respectivas entidades y municipios que comparten. Se menciona el Estado de Hidalgo porque contribuye con un municipio a la ZMVM.
} 
las condiciones de selección definidas pero que no definen el perfil de los municipios que integran la zona metropolitana referida, b) el desface de la información comparada se ha establecido anteriormente y será hasta el momento de tener los datos censales del año 2020 que se pueda actualizar.

La cantidad de población se presenta en números absolutos y corresponde a la Población Económicamente Activa Ocupada (PEA-O). De esta manera es la Zona Metropolitana de la Ciudad de México la que ocupa el primer lugar en tamaño poblacional al estar integrada por 76 municipios de tres entidades, con una PEA-O que oscilaba en los 8.8 millones de sujetos (Tabla 3).

Tabla 3: Ubicación del lugar de trabajo de las Población Económicamente Activa-Ocupada de tres Zonas Metropolitanas de México

\begin{tabular}{lrrrr}
\hline PEA - Ocupada & Total & $\begin{array}{r}\text { Trabaja en el } \\
\text { mismo municipio } \\
\text { (PEAO) }\end{array}$ & $\begin{array}{r}\text { Trabaja en otro } \\
\text { municipio } \\
\text { (PEAO) }\end{array}$ & $\begin{array}{r}\text { Trabaja en otra } \\
\text { entidad } \\
\text { (PEA-OC) }\end{array}$ \\
\hline ZM Valle de México & $8^{\prime} 797,211$ & $57.1 \%$ & $25.4 \%$ & $17.5 \%$ \\
ZM Guadalajara & $2^{\prime} 060,095$ & $70.8 \%$ & $27.7 \%$ & $1.6 \%$ \\
ZM Toluca & 836,115 & $58.6 \%$ & $34.6 \%$ & $6.8 \%$ \\
\hline
\end{tabular}

Fuente: Encuesta Intercensal de 2015 (INEGI).

$\mathrm{Al}$ inspeccionar el análisis propuesto, resalta que son las correlaciones del medio 1 y condición 1 (personas que laboran en el mismo municipio que radican y se desplazan en camión, taxi o colectivo) en donde todos los valores son positivos y significativos al 0.01 , lo cual es congruente con el autor (Tarachini y Cats, 2020). Esta condición no se repite y sugiere que el uso de transporte colectivo se encontraría altamente asociado a los niveles de infección por SARS-CoV-2 como de fallecidos por esta causa. Este resultado podría estar explicado en alguna medida porque la mayor parte de la población examinada manifestó laborar dentro del mismo municipio de residencia y el caso más extremo es Guadalajara con un 70.8 por ciento de la población residente en su Zona Metropolitana (Tabla 3). ${ }^{8}$ Esta relación no se distingue en los tiempos de traslado mismos que no son reportados en las correlaciones (Tabla 4 y Tabla 6).

Nuevamente y dentro del análisis de las respectivas correlaciones, se destaca la fuerte participación de la población de la Zonas Metropolitana de la Ciudad de México que se traslada en bicicleta. Todos los resultados

\footnotetext{
${ }^{8}$ Para el caso de la Zona Metropolitana de Guadalajara no se registran casos del medio de transporte 2, metro, Metrobús o tren ligero. El municipio de Guadalajara sí cuenta con este tipo de transporte pero la correlación lineal no ejecuta el cálculo si no hay datos en todos los municipios.
} 
de las correlaciones son negativos, excepto los contagios de los que trabajan en el mismo municipio, y lo único que indica esta serie de resultados, los cinco restantes, es que hay una correlación negativa y con nivel de significancia de 0.01 en tres de los resultados.

Tabla 4: Tiempo de traslado al lugar de trabajo de la Población Económicamente Activa-Ocupada de tres zonas metropolitanas de México, 2015

\begin{tabular}{|c|c|c|c|c|c|}
\hline PEA-Ocupada & Total & $\begin{array}{l}\text { Tarda hasta } \\
15 \text { minutos }\end{array}$ & $\begin{array}{r}\text { Tarda de } 16 \\
\text { a } 30 \text { minutos } \\
\end{array}$ & $\begin{array}{r}\text { Tarda de } 31 \\
\text { minutos a } 1 \text { hora } \\
\end{array}$ & $\begin{array}{r}\text { Tarda de } \\
1 \text { a } 2 \text { horas } \\
\end{array}$ \\
\hline ZM Valle de México & $8 ' 797,211$ & $18.1 \%$ & $22.2 \%$ & $22.8 \%$ & $16.3 \%$ \\
\hline ZM Guadalajara & $2^{\prime} 060,095$ & $21.7 \%$ & $25.6 \%$ & $26.8 \%$ & $11.2 \%$ \\
\hline ZM Toluca & 836,115 & $20.0 \%$ & $27.9 \%$ & $24.7 \%$ & $10.8 \%$ \\
\hline
\end{tabular}

Fuente: Encuesta Intercensal de 2015 (INEGI).

Es probable que el uso de este medio, la bicicleta, se encuentre altamente asociado a la disminución de la población que trabaja en otro municipio como se puede observar en las respectivas participaciones porcentuales en este medio, 3.3 por ciento la Zona Metropolitana del Valle de México, 3.2 Guadalajara y 5.1 Toluca (Tabla 5).

Tabla 5: Medio de traslado al lugar de trabajo de la Población Económicamente Activa-Ocupada de tres Zonas Metropolitanas de México

\begin{tabular}{lrrr}
\hline PEA-Ocupada & ZM Valle & ZM & ZM \\
& de México & Guadalajara & Toluca \\
\hline Total & $8{ }^{\prime} 797,211$ & $2{ }^{\prime} 060,095$ & 836,115 \\
En Camión, Taxi, Combi o Colectivo & $43.7 \%$ & $38.2 \%$ & $41.8 \%$ \\
En Metro, Metrobús o Tren ligero & $3.1 \%$ & $0.6 \%$ & $0.0 \%$ \\
Vehículo particular (automóvil, & $17.0 \%$ & $27.5 \%$ & $20.1 \%$ \\
camioneta o moto) & $2.0 \%$ & $5.0 \%$ & $2.6 \%$ \\
En Transporte laboral & $3.3 \%$ & $3.2 \%$ & $5.1 \%$ \\
En Bicicleta & $14.8 \%$ & $13.1 \%$ & $16.0 \%$ \\
Caminando & &
\end{tabular}

Fuente: Encuesta Intercensal de 2015 (INEGI).

Como ya se ha mencionado, los medios de transporte como metro, Metrobús o tren ligero participan sólo en dos de las zonas metropolitanas consideradas, Valle de México y Guadalajara, pero se encuentran sujetas al traslado experimentado por los sujetos en cuestión. A pesar de ello, el valor 
de la $r$ que reportan para los sujetos que trabajan en otro municipio es 0.59 y 0.49 con respecto a fallecidos y contagiados, ambos casos con un nivel de significancia de 0.01 .

Otro resultado de este análisis son dos correlaciones especialmente elevadas, $r$ de 0.93 y 0.96 que corresponden a quienes trabajan en el mismo municipio de la Zona Metropolitana de Toluca y utilizan vehículo particular y moto (Tabla 6). Este resultado se podría encontrar influenciado por la cantidad de población que comparte este tipo de transporte u otras variables que no son detectadas en la fuente usada.

Finalmente se destaca que en la Tabla 6 el número de celdas que reportan correlación con el número de fallecidos difiere poco con respecto al número de contagiados si a significancias de 0.01 o con 0.05 nos referimos.

\section{Conclusiones}

El impacto que ha tendido el uso del transporte colectivo en los volúmenes de fallecimientos e infectados dentro de las tres Zonas Metropolitanas es notorio. Esta condición se expresa no únicamente en los elevados valores del valor $r$, de los seis sólo en dos de ellos es menor a 0.8 .

Por otro lado, es igualmente visible que el uso de la bicicleta como transporte hacia las actividades laborales reporta valores negativos y altamente significativos.

Se debe reconocer la importancia de la población bajo condiciones de movilidad laboral con el objeto de precisar su cuantificación como su perfil socioeconómico. Frecuentemente se deja que los actores privados se hagan cargo de la solución de los problemas de movilidad afectando con ello las condiciones de traslado dado que priva el criterio de la utilidad.

Los resultados presentados en este documento ilustran una parte del comportamiento sobre el uso de distintos tipos de transporte utilizado para desplazarse con fines laborales. Bajo el análisis presentado, el uso de la bicicleta muestra un recurso que de examinarse con más detalle proporcionaría elementos para la elaboración de políticas sociales tendientes a estimular su uso. Una alternativa es la construcción de vías que conecten los espacios residenciales y laborales con la evaluación previa obviamente. El estímulo de la bicicleta con fines recreativos es una opción viable, pero se debe evaluar el posible impacto de esta decisión. 
Movilidad laboral urbana y Covid-19: los casos de tres zonas metropolitanas de México / J.A. SOBERÓN MORA et al.

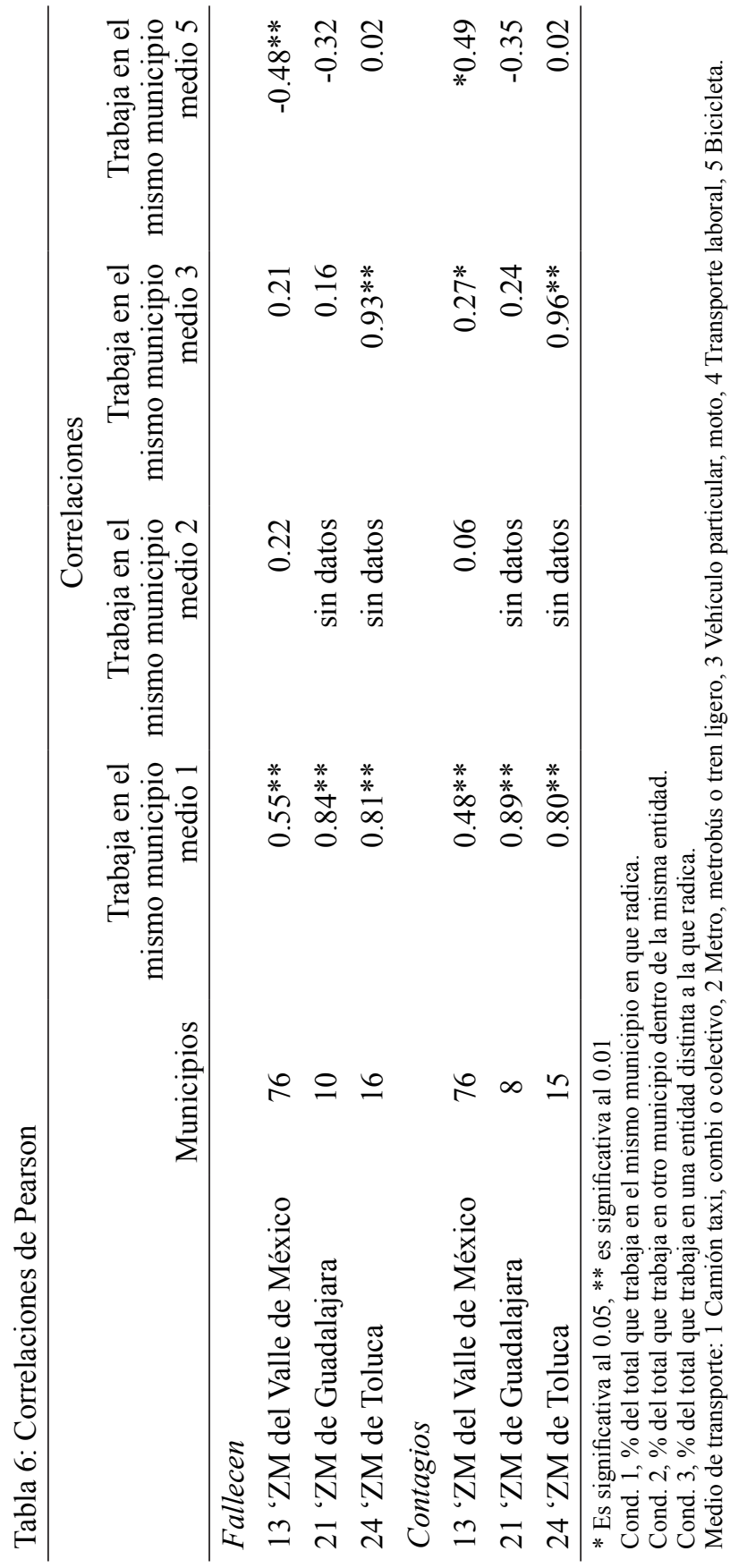




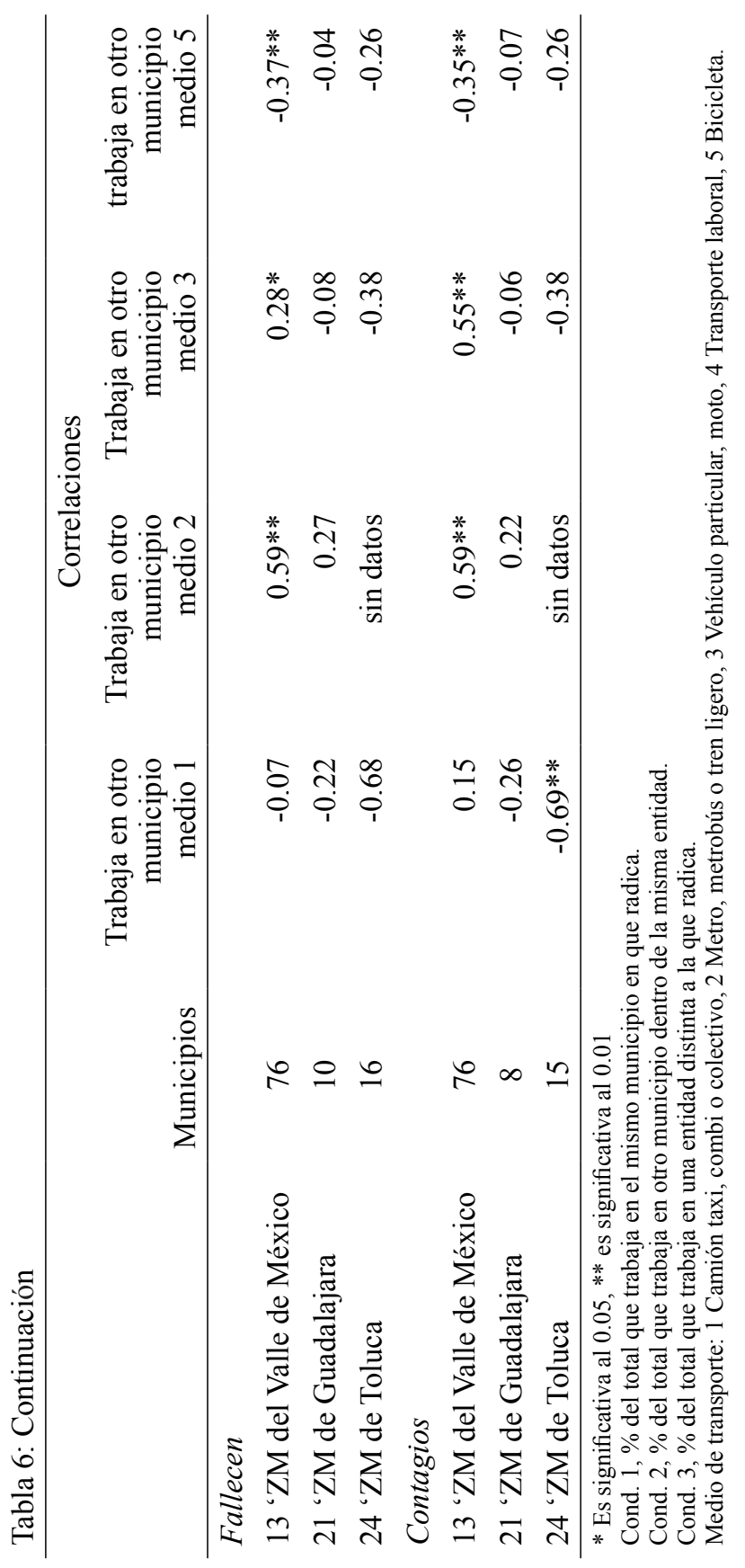


Movilidad laboral urbana y Covid-19: los casos de tres zonas metropolitanas de México / J.A. SOBERÓN MORA et al.

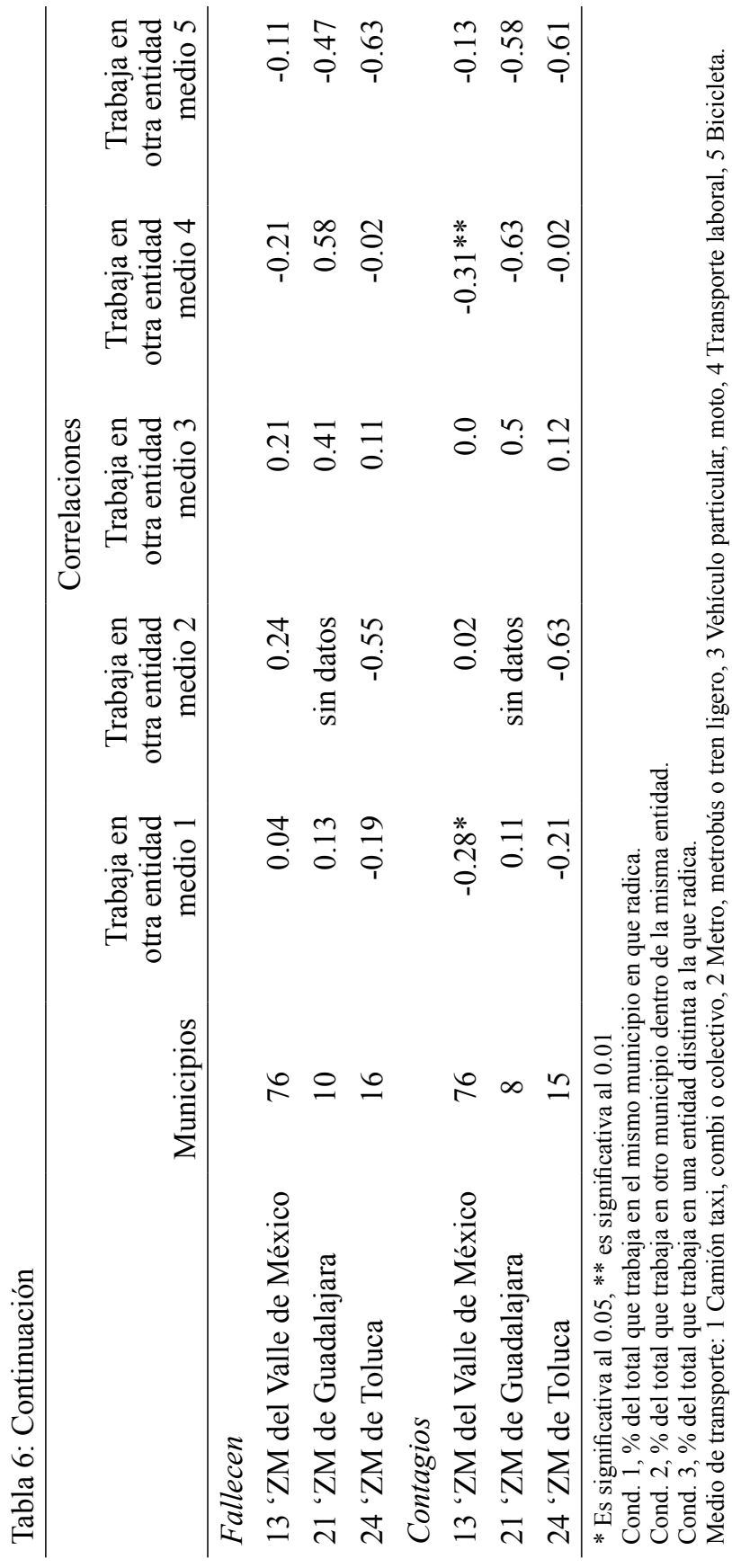


Al proponer la evaluación de las opciones para el uso de bicicleta, o cualquier otro medio, es necesario estimar los volúmenes respectivos sobre el traslado, las distancias, los puntos, esto es, la posible selección entre uno de ellos principalmente habitacional y otro con prevalencia de actividad comercial, industrial o incluso simultanea y el perfil de los usuarios respectivos, tal y como se ha hecho durante años. ¿Qué significa? La población laboral ya está y ya se mueve entre dos puntos, entonces queda la posibilidad de estimar la factibilidad del cambio de medios de transporte opcionales como en este caso el uso de la bicicleta.

Cuando mencionamos que la población se mueve entre dos puntos, en realidad los puntos son múltiples. Por un lado, la multiplicidad de puntos en una línea, en donde el acceso a una vía puede ser en cualquiera de los sitios intermedios de esa línea, por el otro la salida puede ser en cualquier momento y no necesariamente al final de esta. En un segundo plano, dentro de una región existe policentrismo y las posibilidades y usos del traslado laboral son tan diversas como la cantidad de población que se mueve dentro de la región con ese fin. Sin embargo queda la opción, como se ha mencionado, de evaluar los movimientos más representativos con la finalidad de seleccionar los más promisorios ya sea por volumen de usuarios, actitud al cambio, evaluación de riesgos, distancia, o distancias medias a cubrir o principales horarios laborales por citar algunos elementos a considerar.

\section{REFERENCIAS BIBLIOGRÁFICAS}

Acaps M., 2020, Covid-19: government measures. https://www.acaps.org/special-report/covid-19-government-measures. Recuperado el 25/03/2020.

Animal político, 2020, EDOMEX. Disponible en https://www.animalpolitico. com/2020/11/chihuahua-nuevas-medidas-restriccion-transporte-comercios/

Bautista Lucca, Cintia, s/f, Decisiones metodológicas en la comparación de fenómenos políticos iberoamericanos. Instituto de Iberoamérica, Universidad de Salamanca, Documentos de Trabajo Juan P21.

Camacho, Marce D., 2015, Estructuración metropolitana de Toluca. Los impactos de la desurbanización en el territorio en el periodo 1990 a 2010, Tesis de Maestría, Universidad Autónoma del Estado de México.

Chávez, Ana María y Guadrrama, Julio, s/f, La región central en transición: tendencias económicas y migratorias a finales del milenio, disponible en https:// speedy.cepal.org/sites/default/files/courses/files/chavez_articulo.pdf (consultado el 31/01/2021).

CONAPO, 2015, Delimitación de las zonas metropolitanas de México, Secretaría de Desarrollo Agrario, Consejo Nacional de Población, Instituto Nacional de Estadística y Geografía, México, primera edición 2018. 
ECDC, 2020, Situation update worldwide, as of 13 April 2020: ECDC; 2020. Disponible en https://www.ecdc.europa.eu/en/geographical-distribution-2019-ncov-cases. Recuperado el 13/04/2020.

Feehan, D. y Ayesha, Mahmud, 2020, "Quantifying population contact patterns in the United States during the Covid-19 pandemia", in Nature Communications, 12:893. Disponible en https://doi.org/10.1038/s41467-021-20990-2 www.nature. com/naturecommunications

Gaceta Oficial de la Ciudad de México, 2020, Órgano de Difusión del Gobierno de la Ciudad de México. Vigésima Primera Época, 1 de abril de 2020, No. 315 Bis.

Garza Merodio, Gustavo, 2020, Zona Metropolitana de Toluca, Disponible en http://www.igg.unam.mx/covid-19/Vista/archivos/atlas/ZMTOL.pdf (consultado el 16/01/2021).

Glass, G. y Stanley, Julian C., 1974, Métodos Estadísticos Aplicados a las Ciencias Sociales, Prentice/Hall, Barcelona, España.

Gobierno de Nuevo León, 2020, https://www.nl.gob.mx/campanas/medidas-preventivas-en-el-transporte-publico-contra-el-covid-19

Granados-Alcantar, José Aurelio, Franco-Sánchez, Laura Myriam, 2017, Migración y movilidad laboral entre las zonas metropolitanas de la región centro de México, Disponible en http://www.scielo.org.mx/scielo.php?script=sci_arttext\&pid=S1405-74252017000100117 (consultado el 15/01/2021).

Hernández Almejo, Rubén y Benitez Villegas, Israel, 2017, Asociaciones entre la movilidad laboral intrametropolitana y distribución espacial de empleo en la Zona Metropolitana de Toluca: una aproximación desde los sectores de uso intensivo del conocimiento, Disponible en https://archivos.juridicas.unam.mx/www/ bjv/libros/11/5392/10.pdf (consultado el 16/01/2021).

Hoyos Castillo, Guadalupe, Rozga Luter, Ryszard y Sánchez Nájera, Rosa María, s/f, Estructura espacial de la movilidad residencia-trabajo en la Zona Metropolitana de Toluca, 2000 - 2015, Disponible en http://ru.iiec.unam.mx/3930/1/242-Hoyos-R\%C3\%B3zga-S\%C3\%A1nchez.pdf (consultado el 15/01/2021).

IMSS, 2020, Fases de contingencia y nivel de propagación del Covid-19, Disponible en http://educacionensalud.imss.gob.mx/es/system/files/Fases-COVID19. pdf

Informador, 2020, https://www.informador.mx/jalisco/Coronavirus-en-Jalisco-Asi-funcionara-el-transporte-publico-con-el-boton-de-emergencia-20201028-0039.html

Jarvis, CI, Van Zandvoort, Kevin, Gimma, Amy, Prem, Kiesha, 2020, “Quantifying the impact of physical distance measures on the transmission of Covid-19 in the UK", in BMC Medicine 18:124, Disponible en https://doi.org/10.1186/s12916020-01597-8

López Vega, Rafael, 2016, "Movilidad espacial de la población en México", en Contexto, No. 62, mayo, CESOP Centro de Estudios Sociales y de Opinión Pública, Cámara de Diputados, México. 
OMS, 2020, https://www.who.int/es/news-room/commentaries/detail/modes-of-transmission-of-virus-causing-covid-19-implications-for-ipc-precaution-recommendations

Potrykowski, Marek y Taylor, Zbigniew, 1984, Geografía del transporte, Ariel, S. A.

Ramírez, B., 2015, “Alcances y dimensiones de la movilidad: aclarando conceptos”, en Ramírez Velázquez, Blanca Rebeca (coord.), Debates y estudios de la movilidad laboral en la región centro del país: alcances y dimensiones desde México, Universidad Autónoma Metropolitana.

Romo, Raúl, 2018, La migración metropolitana en México: predominante y estable, disponible en https:/www.gob.mx/cms/uploads/attachment/file/491895/ p108-p123.pdf (consultado el 16/01/2021).

Sánchez Salazar, María Teresa y Suárez Lastra, Manuel, s/f, Zona Metropolitana de la Ciudad de México, http://www.igg.unam.mx/covid-19/Vista/archivos/atlas/ ZMValleMexico.pdf (consultado el 16/01/2020).

Secretaría de Salud, 2020, Base de datos Covid-19, disponible en https://datos. gob.mx/busca/dataset/informacion-referente-a-casos-covid-19-en-mexico.

Suárez, Manuel, Galindo, P. Carlos, Rosales, Ana Rosa y Salvador, Luis Enrique, 2020, Territorio y vulnerabilidad ante Covid-19 en México. Las Ciencias Sociales y el coronavirus, COMECSO, México.

Tarachini, A. y Catz, Odet, 2020, "Covid-19 and Public Transportation: Current Assessment, Prospects, and Research Needs", in Journal of Public Transportation scholarcommons, vol. 22 No. 1 pp. 1-21.

UNAM, 2015, Movilidad interna, Capitulo 2. Disponible en https://archivos.juridicas.unam.mx/www/bjv/libros/11/5299/4.pdf (consultado el 15/01/2021).

Vargas Espinosa, Victor Hugo, Romero Hernández, David, Castillo-Aja, Rocío, Corona, Néstor y Ramírez Herrera, María Teresa, s/f, Zona Metropolitana de Guadalajara, disponible en http://www.igg.unam.mx/covid-19/Vista/archivos/atlas/ ZMGDL.pdf (consultado el 16/01/2020).

Zhang J., 2020, “Age profile of susceptibility, mixing, and social distancing shape the dynamics of thenovel coronavirus disease 2019 outbreak in China", in Epidemiology. Disponible en https://doi.org/10.1101/2020.03.19.20039107

\section{RESUMEN CURRICULAR DE LOS AUTORES}

José Antonio Soberón Mora

Licenciado en Psicología Social, Maestro en Demografía y Doctor en Urbanismo por la Universidad Autónoma del Estado de México. Autor y coautor de diversos capítulos de libro. Actualmente desarrolla el proyecto: Crecimiento Económico y Migración Interna en las Zonas Metropolitanas 
de México (1990-2010). Profesor investigador de la Universidad Autónoma del Estado de México.

Dirección electrónica: josesoberon2004@yahoo.com

Registro ORCID: https://orcid.org/0000-0003-2812-100x

\section{Juan Gabino González Becerril}

Maestro en Estudios de Población por El Colegio de la Frontera Norte. Es profesor-investigador de tiempo completo en el Centro de Investigación y Estudios Avanzados de la Población de la Universidad Autónoma del Estado de México (CIEAP/UAEM) y es director de la revista Papeles de Población.

Dirección electrónica: gonzalezg2012@hotmail.com

\section{Rodrigo Aguilar Zepeda}

Doctor en Estudios de Población por El Colegio de México. Sus principales líneas de investigación son: migración de retorno a México (caracterización sociodemográfica), migración de retorno de niños y jóvenes a México y su relación con la inserción escolar, migración de retorno en hogares y la reestructuración de estos; así como el tema de integración en contextos de retorno. Ha publicado artículos y capítulos de libro relacionados con sus líneas de investigación, entre ellos ¿Y si quiero regresar a mi país? Niños estadounidenses en escuelas de Cuernavaca (2018) y Migración de retorno infantil y juvenil en México: cambios y desafios educativos (2019). En la actualidad es profesor investigador de Tiempo Completo en el Centro de Investigación y Estudios Avanzados de la Población en la Universidad Autónoma del Estado de México. Actualmente es candidato al Sistema Nacional de Investigadores (S N I).

Dirección electrónica: rodro_az@yahoo.com.mx

\section{Eduardo Andrés Sandoval Forero}

Antropólogo, Maestro en Estudios Latinoamericanos, Doctor en Sociología por la Universidad Nacional Autónoma de México (UNAM). Miembro del Sistema Nacional de Investigadores de México desde 1995 (Nivel II). Profesor invitado de universidades de Estados Unidos, América del Sur, España e Italia. Es profesor del curso Migración y codesarrollo en la Cátedra UNESCO, del posgrado de Estudios para la Paz y el Desarrollo, en la Universitat Jaume I, España. Autor de varios artículos, capítulos y libros sobre migración, diversidad cultural y grupos étnicos. Líder del Cuerpo Académico en Migración Interna e Internacional del CIEAP-UAEM. Su 
mas reciente libro es Educación para la paz integral. Memoria, Interculturalidad y decolonialdad.

Dirección electrónica: forerosandoval@gmail.com

Registro ORCID: https://orcid.org/000-0003-159-7588

Artículo recibido el 21 de enero de 2021 y aprobado el 5 de febrero de 2021. 